\title{
Validity of bone marker measurements for monitoring response to bisphosphonate therapy with zoledronic acid in metastatic breast cancer
}

\author{
BAHRIYE AKTAS $^{1}$, SABINE KASIMIR-BAUER $^{1}$, NILS LEHMANN $^{2}$, RAINER KIMMIG $^{1}$ and MITRA TEWES ${ }^{3}$ \\ ${ }^{1}$ Department of Gynecology and Obstetrics, ${ }^{2}$ IMIBE, Institute for Medical Informatics, Biometry and Epidemiology; \\ ${ }^{3}$ Department of Medical Oncology, West German Cancer Center, University Hospital Essen, \\ University of Duisburg-Essen, D-45122 Essen, Germany
}

Received July 23, 2012; Accepted September 17, 2012

DOI: 10.3892/or.2013.2409

\begin{abstract}
Bone is the most common site of metastasis in breast cancer. Detection relies on imaging technology which is costly and can only be performed to a certain degree. Bone markers are non-invasive, inexpensive and may potentially serve as predictive and prognostic surrogate endpoints in detecting bone metastases and response to bisphosphonates. This study analyzed the value of the serum bone turnover markers PINP and ICTP for bone metastases in metastatic breast cancer patients receiving zoledronic acid. The results were compared with the serum levels of CEA and CA 15-3, and analyzed with respect to the number of bone metastases as well as clinical response. Forty patients with confirmed bone metastases who received chemotherapy and/or hormonal therapy and zoledronic acid i.v. q4 weeks participated in the present study. Blood $(5 \mathrm{ml})$ was collected at the start of the study and $\mathrm{q} 3$ months for a period of one year for the analysis of PINP, ICTP, CEA and CA 15-3 using radioimmunoassays and ELISA, respectively. Imaging of bone metastases was performed at the same time points. In 29 out of 40 patients, more than 3 bone metastases were confirmed by imaging and 11 out of 40 patients presented with 3 or less. At the start of the study, the median value for ICTP was $6 \mu \mathrm{g} / 1$ and for PINP $58.7 \mu \mathrm{g} / \mathrm{l}$. At the end of the study the median values were $4.5 \mu \mathrm{g} / 1$ for ICTP and $21 \mu \mathrm{g} / 1$ for PINP. When patients were stratified into responders and non-responders, a decrease
\end{abstract}

Correspondence to: Dr Bahriye Aktas, Department of Gynecology and Obstetrics, University of Duisburg-Essen, Hufelandstrasse 55, D-45122 Essen, Germany

E-mail: bahriye.aktas@uk-essen.de

Abbreviations: $\mathrm{MBC}$, metastatic breast cancer; $\mathrm{BM}$, bone metastases; PINP, aminoterminal procollagen propeptide of type I collagen; ICTP, carboxyterminal telopeptide of type I collagen; PD, progressive disease; PR, partial remission; SD, stable disease; $\mathrm{CR}$, complete remission; ZOL, zoledronic acid; BP, bisphosphonate; CEA, carcinoembryonic antigen; CA 15-3, cancer antigen 15-3; IQR, interquartile range

Key words: bisphosphonate, bone marker, therapy monitoring, metastatic breast cancer in both PINP $(\mathrm{P}<0.0001)$ and ICTP $(\mathrm{P}=0.048)$ was observed for the responders, while the level of ICTP $(\mathrm{P}=0.02)$ increased for the non-responders. Serum PINP and ICTP concentrations were significantly different when patients were stratified into groups of those having more than 3 bone metastases and 3 or less, respectively $(\mathrm{P}<0.05)$. CEA and CA $15-3$ levels did not differ with respect to the number of bone metastases, while the tumor marker levels determined at the end of the study significantly distinguished responders from non-responders $(\mathrm{P}=0.002$ and $\mathrm{P}=0.004)$. In conclusion, in contrast to serum tumor markers, the determination of PINP and ICTP allows inferences to the number of bone metastases and appears to be a useful tool for prediction and monitoring metastatic breast cancer patients undergoing bisphosphonate therapy with zoledronic acid for the treatment of bone metastases.

\section{Introduction}

Bone metastases (BM) are a frequent event in breast cancer and are difficult to detect. The assessment of skeletal metastases primarily depends on imaging techniques. Routinely, bone scintigraphy is used for detection of BM. Although bone scintigraphs have high sensitivity, they lack specificity in the detection of skeletal metastases $(1,2)$. Thus, additional available imaging techniques such as radiography, computer tomography or magnetic resonance imaging are necessary to verify the bone scan, and these techniques are highly expensive, particularly for monitoring therapeutic response of skeletal metastases during palliative systemic therapy.

Malignant bone disease is associated with increased levels of bone resorption markers $(3,4)$. Osteoblasts and osteoclasts are affected by malignant cells in skeletal metastases causing increased number, activity and survival of these bone remodelling cells, a phenomenon known as the vicious cycle (5). In this regard, series of biochemical serum markers of bone turnover, such as aminoterminal procollagen propeptide of type I collagen (PINP) and carboxyterminal telopeptide of type I collagen (ICTP), have been evaluated for the early detection of skeletal metastases $(6,7)$.

Bisphosphonates inhibit osteoclast-mediated osteolysis and reduce bone marker levels $(8,9)$. Zoledronic acid (ZOL) 
reduces the risk of skeletal-related events and suppresses levels of bone turnover markers in patients with multiple myeloma or bone metastases from solid tumors, including breast cancer $(10,11)$. Assuming that bone metastasis requires a modification of bone formation and resorption, it seems likely that measurement of bone markers during palliative therapy in metastatic breast cancer (MBC) patients could be useful to monitor the therapeutic response. In contrast to imaging techniques, bone marker measurements are easy to perform, minimally invasive and inexpensive.

Therefore, the objective of this study was to assess PINP and ICTP levels in MBC patients receiving standard systemic therapy with ZOL over the course of time for one year and analyze these findings in relation to response rates assessed by imaging techniques and to the serum levels of tumor markers CEA and CA 15-3.

\section{Patients and methods}

Patients and study design. This prospective study included MBC patients with bone metastases. The study was conducted at the Department of Obstetrics and Gynecology at the University Hospital in Essen. Forty MBC patients (mean age 60 years; range, 36-85) with confirmed BM who received systemic therapy including chemotherapy or hormonal therapy and ZOL $4 \mathrm{mg}$ i.v. q4 weeks participated in our study (Table I).

Blood (5 ml) was collected at the start of the study and every 3 months for one year for the analysis of levels of PINP, ICTP, CEA and CA 15-3 using radioimmunoassays and ELISA technique. Imaging of $\mathrm{BM}$ was performed at the same time points.

The trial was initiated after the approval of the Institutional Ethics Committee Review Board.

Eligibility criteria. The eligibility criteria were as follows: histologically confirmed breast cancer disease; patient age $\geq 18$ years; measurable or evaluable BM; predicted life expectancy $\geq 3$ months; Eastern Cooperative Oncology Group (ECOG) scores for performance status of $0-2$; no severe uncontrolled co-morbidities or medical conditions; no second malignancies.

Patients had either a relapse of breast cancer diagnosed years before and were to start a new systemic therapy or they had a documented progressive breast cancer before receiving a new endocrine, chemo- or experimental therapy in combination with ZOL. Prior adjuvant treatment, radiation or any other treatment of metastatic disease were permitted. ZOL in the metastatic setting was not allowed. Further exclusion criteria were other malignancies except breast cancer.

Response criteria. Before starting a new treatment, patients underwent an evaluation of metastatic sites, particularly BM by bone scintigraphy, X-ray and/or computer tomography. Blood samples were collected for laboratory evaluations, including assaying of plasma CEA and CA 15-3 levels as well as for the assessment of PINP and ICTP. Re-evaluation of disease status was carried out using the same techniques every 12 weeks for one year.

Response to therapy was evaluated according to the Response Evaluation Criteria in Solid Tumors (RECIST); complete response (CR), disappearance of all target lesions; partial response (PR), at least $30 \%$ decrease in the sum of the
LD (longest diameter) of target lesions, taking as reference the baseline sum of the LDs; progressive disease (PD), at least $20 \%$ increase in the sum of the LDs of the target lesions, taking as reference the smallest sum of the LDs recorded since the treatment started or the appearance of one or more new lesions; stable disease (SD), neither sufficient shrinkage to qualify for PR nor sufficient increase to qualify for PD, taking as reference the smallest sum of the LDs since the treatment started.

Sampling of serum. Blood $(2 \times 5 \mathrm{ml})$ was collected with an S-Monovette ${ }^{\circledR}$ (Sarstedt AG \& Co.) at the start of the study and every 3 months for one year for the analysis of PINP, ICTP, CEA and CA 15-3. The samples were processed immediately and the serum was stored at $-80^{\circ} \mathrm{C}$ until further examination.

Determination of markers of bone turnover. PINP and ICTP were assayed by radioimmunoassay (RIA) (Orion Diagnostica, Oulunsalo, Finland). The reference range for PINP was $19-84 \mu \mathrm{g} / 1$ and the reference range for ICTP was $1.8-5 \mu \mathrm{g} / 1$. The samples for bone marker measurement were sent to and analyzed by an extern laboratory (Department of Medical Microbiology and Infectious Diseases, Medical Care Centre, Dr Stein and colleagues, Mönchengladbach, Germany).

Determination of serum tumor markers. CEA and CA 15-3 were determined using the Elecsys CEA/CA 15-3 immunoassays (Roche, Mannheim, Germany) for the quantitative determination in human serum and plasma. The serial measurement of CEA/CA 15-3 is intended to aid in the management of cancer patients. These assays were performed in the central laboratory of our University Hospital on Cobas ${ }^{\circledR}$ immunoassay analyzers according to the manufacturer's instructions. The central laboratory has a valid certification for the performance of these assays following international guidelines. The reference range for CEA was $<5 \mathrm{ng} / \mathrm{ml}$, and the reference range for CA $15-3$ was $<35 \mathrm{U} / \mathrm{ml}$.

Statistical consideration. Statistical analyses were carried out using SAS v. 9.2 (SAS Institute Inc., Cary, NC). Differences in continuous parameters between the groups were tested by the non-parametric Mann-Whitney $U$ test, and changes within groups were evaluated using the Wilcoxon sign rank test. Differences in proportions were tested using Chi-square statistics or Fisher's exact test, as appropriate. A P-value of $<0.05$ was regarded as statistically significant. The statistical significance of the differences in receiver-operating characteristic (ROC) analysis was determined using the method developed by DeLong, DeLong and Clarke-Pearson.

\section{Results}

Characteristics of the study population. Patient ages ranged from 36 to 85 years (median 60). A total of 40 patients were enrolled since January 2008. Ten out of 40 (25\%) were premenopausal and 30 out of $40(75 \%)$ were postmenopausal. The patient characteristics are documented in Table I. Thirtyfour out of 40 patients $(85 \%)$ had a primary metastatic breast cancer, 4 out of $40(10 \%)$ received second-line therapy and 2 out of $40(5 \%)$ received third-line or more in the metastatic setting. Most patients $(60 \%)$ had ductal breast cancer. 
Table I. Patient characteristics.

\begin{tabular}{|c|c|c|}
\hline & $\begin{array}{c}\text { Study group } \\
\mathrm{n}(\%)\end{array}$ & $\begin{array}{c}\text { Comparison group } \\
\mathrm{n}(\%)\end{array}$ \\
\hline Overall & $40(100)$ & $11(100)$ \\
\hline \multicolumn{3}{|l|}{ Histology } \\
\hline Ductal & $24(60.0)$ & $6(54.5)$ \\
\hline Lobular & $11(27.5)$ & $4(36.4)$ \\
\hline Others & $5(12.5)$ & $1 \quad(9.1)$ \\
\hline \multicolumn{3}{|l|}{ ER status } \\
\hline Negative & $6(15)$ & $4(36.4)$ \\
\hline Positive & $34(85)$ & $7(63.6)$ \\
\hline \multicolumn{3}{|l|}{ PR status } \\
\hline Negative & $16(40)$ & $6(54.5)$ \\
\hline Positive & $24(60)$ & $5(45.5)$ \\
\hline \multicolumn{3}{|l|}{ HER 2 status } \\
\hline Negative 1 & $33(82.5)$ & $9(81.8)$ \\
\hline Positive 2 & $6(15.0)$ & $1 \quad(9.1)$ \\
\hline Unknown 3 & $1 \quad(2.5)$ & $1 \quad(9.1)$ \\
\hline \multicolumn{3}{|l|}{ Metastatic site } \\
\hline Bone & $21(52.5)$ & $0 \quad(0)$ \\
\hline Visceral and bone & $19(47.5)$ & $11(100)$ \\
\hline \multicolumn{3}{|l|}{ Metastatic site } \\
\hline One site & $7(17.5)$ & $2(9.1)$ \\
\hline Multiple sites & $33(82.5)$ & $9(81.8)$ \\
\hline \multicolumn{3}{|l|}{$\begin{array}{l}\text { No. of bone } \\
\text { metastases }\end{array}$} \\
\hline$\leq 3$ & $11(27.5)$ & $0(0)$ \\
\hline$\geq 3$ & $29(72.5)$ & $0(0)$ \\
\hline \multicolumn{3}{|l|}{ Therapeutic setting } \\
\hline First-line & $34(85)$ & $6(54.5)$ \\
\hline Second-line & $4(10)$ & $3(18.2)$ \\
\hline Third-line or more & $2(5)$ & $2(18.2)$ \\
\hline \multicolumn{3}{|l|}{ Clinical response } \\
\hline $\mathrm{CR} / \mathrm{PR} / \mathrm{SD}$ & $30(75)$ & \\
\hline PD & $10(25)$ & \\
\hline
\end{tabular}

CR, complete remission; $\mathrm{PR}$, partial remission; $\mathrm{SD}$, stable disease; $\mathrm{PD}$, progressive disease.

Moderately and poorly differentiated tumors were predominant (data not shown). Twenty-four out of 40 (76.5\%) of the primary tumors were ER- and PR-positive, respectively, and only $15 \%$ (6 out of 40) had an overexpression of HER2 (Dako score $3+)$. Nineteen out of 40 patients $(47.5 \%)$ had visceral and non-visceral metastases, 21 out of 40 patients $(52.5 \%)$ only BM. In 29 out of 40 patients $(72.5 \%)>3 \mathrm{BM}$ were confirmed by imaging and 11 out of 40 patients $(27.5 \%)$ presented with $\leq 3$ BM. Patients received different chemotherapeutic treatments including anthracyclines, taxanes, capecitabine, vinorelbine and 5-FU (data not shown). After starting measurements of PINP, ICTP and tumor markers, patients underwent individual chemotherapeutic or hormonal treatment depending on their pretreatment and the addition of ZOL.

The control group consisted of 11 patients who had visceral metastases only and no BM. Median age of the control group was $66(43-85)$ years.

Stratification of results. In total, 40 patients were monitored for PINP, ICTP, CA 15-3 and CEA during chemotherapy over a time period of 12 months. Blood samples were collected and imaging techniques were performed every three months. Based on the total number of collected blood samples, only baseline and the data evaluated at the end of the study were statistically analyzed. The results for PINP and ICTP were analyzed in relation to clinical response (stratified into responders and non-responders), the number of BM ( $\leq 3$ or $>3$ ) and tumor markers. Responders were defined as patients with complete remission (CR), partial remission (PR) and stable disease (SD). Non-responders included patients with progressive disease (PD).

Comparison group. The median value of ICTP was $5.44 \mu \mathrm{g} / \mathrm{l}$ (IQR 3-12.8) and the median value of PINP was $55.67 \mu \mathrm{g} / \mathrm{l}$ (IQR 17.9-112.3). The CA 15-3 level was $123.27 \mathrm{U} / \mathrm{ml}$ (IQR 19-682) and the CEA level was $9.23 \mathrm{ng} / \mathrm{ml}$ (IQR 1.5-61.8).

Correlation of PINP and ICTP with number of BM. The relationship among PINP, ICTP and the number of BM is shown in Fig. 1. Twenty-nine patients had $>3 \mathrm{BM}, 11$ patients has $\leq 3$. Serum PINP and ICTP concentrations were significantly different when patients were stratified into groups according to whose with >3 BM (median value ICTP, 6.2 $\mu \mathrm{g} / \mathrm{l}$; IQR 4.8-16.8 and PINP, 66.6 $\mu \mathrm{g} / \mathrm{l}$; IQR 49.4-221) and $\leq 3 \mathrm{BM}$ (median value ICTP, $4 \mu \mathrm{g} / \mathrm{l}$; IQR 3-6.1 and PINP, $50.4 \mu \mathrm{g} / \mathrm{l}$; IQR 35.6-54.2).

Correlation of PINP and ICTP with the response to therapy. The levels of PINP and ICTP, determined at the start and the end of the follow-up period, were analyzed with respect to clinical follow-up results. At the start of the study, the median value for ICTP was $6 \mu \mathrm{g} / \mathrm{l}$ (IQR 3.9-12.7) and the median value for PINP was $58.7 \mu \mathrm{g} / \mathrm{l}$ (IQR 43.5-164). Thirty out of 40 patients showed response to therapy, and 10 out of 40 patients had progressive disease. The median value of ICTP at the start of the study for the responders was $5.1 \mu \mathrm{g} / \mathrm{l}$ (IQR 3.6-7.2) vs. $20.4 \mu \mathrm{g} / \mathrm{l}$ (IQR 6.9-43.9) for non-responders. The median value of PINP at the start of the study for the responders was $50.4 \mu \mathrm{g} / \mathrm{l}$ (IQR 36.4-66.6) vs. $221 \mu \mathrm{g} / 1$ (IQR 59-250) for the non-responders $(\mathrm{P}=0.0003$ for ICTP and $\mathrm{P}=0.005$ for PINP) (Fig. 2). At the end of the study, the mean value of ICTP was $4.1 \mu \mathrm{g} / \mathrm{l}$ (IQR 3.1-6) for the responders vs. $50 \mu \mathrm{g} / 1$ (IQR 18.4-50) for nonresponders. In contrast, the median value of PINP at the study end was 17.3 $\mu \mathrm{g} / 1$ (IQR 10.8-25.6) for responders vs. $250 \mu \mathrm{g} / \mathrm{l}$ (IQR 101.2-250) for non-responders ( $\mathrm{P}=0.0004$ for ICTP and $\mathrm{P}=0.0007$ for PINP) (Fig. 2). Changes in expression levels of the markers from the start to the end of the study were significantly different from zero for PINP $(\mathrm{P}<0.0001)$ and ICTP $(\mathrm{P}=0.048)$ (decrease) for responders and ICTP $(\mathrm{P}=0.02)$ for non-responders (increase, Fig. 3).

Correlation between tumor markers and number of bone metastases. As shown in Fig. 4A no significant differences were 

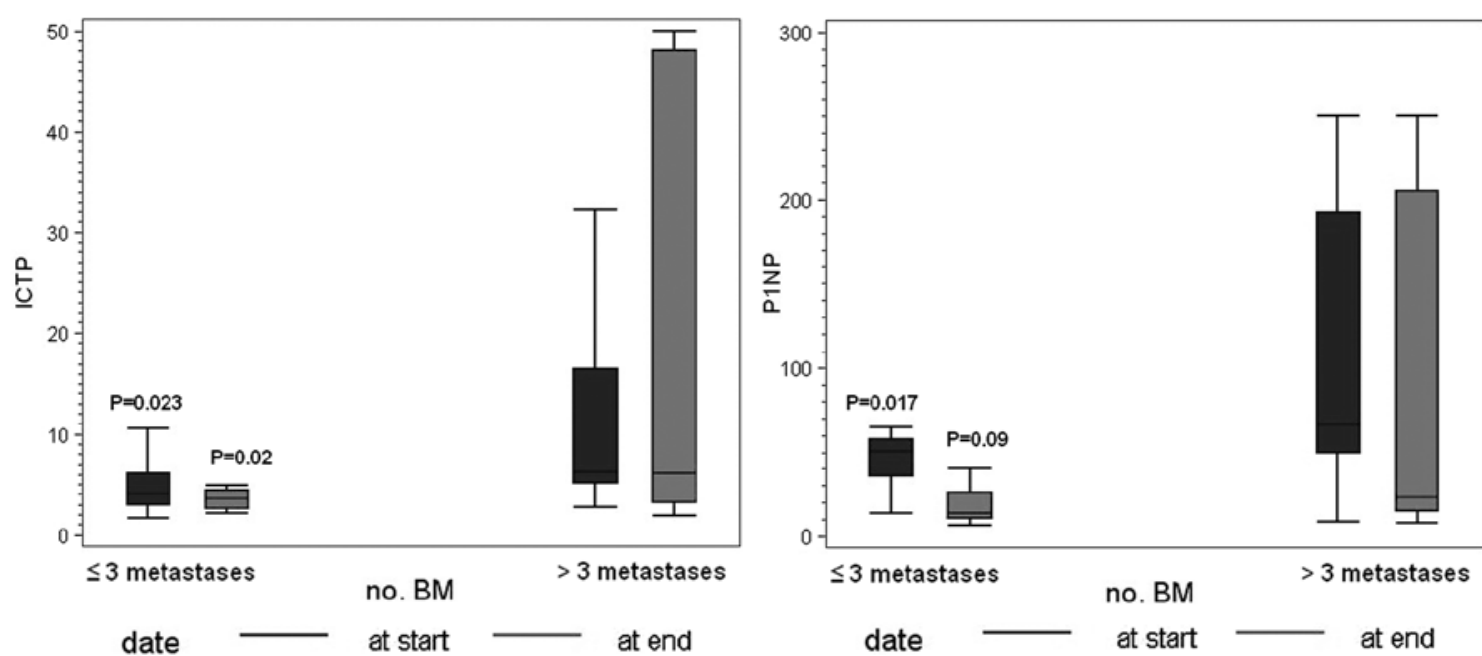

Figure 1. Correlation of levels of PINP/ICTP and the number of bone metastases.
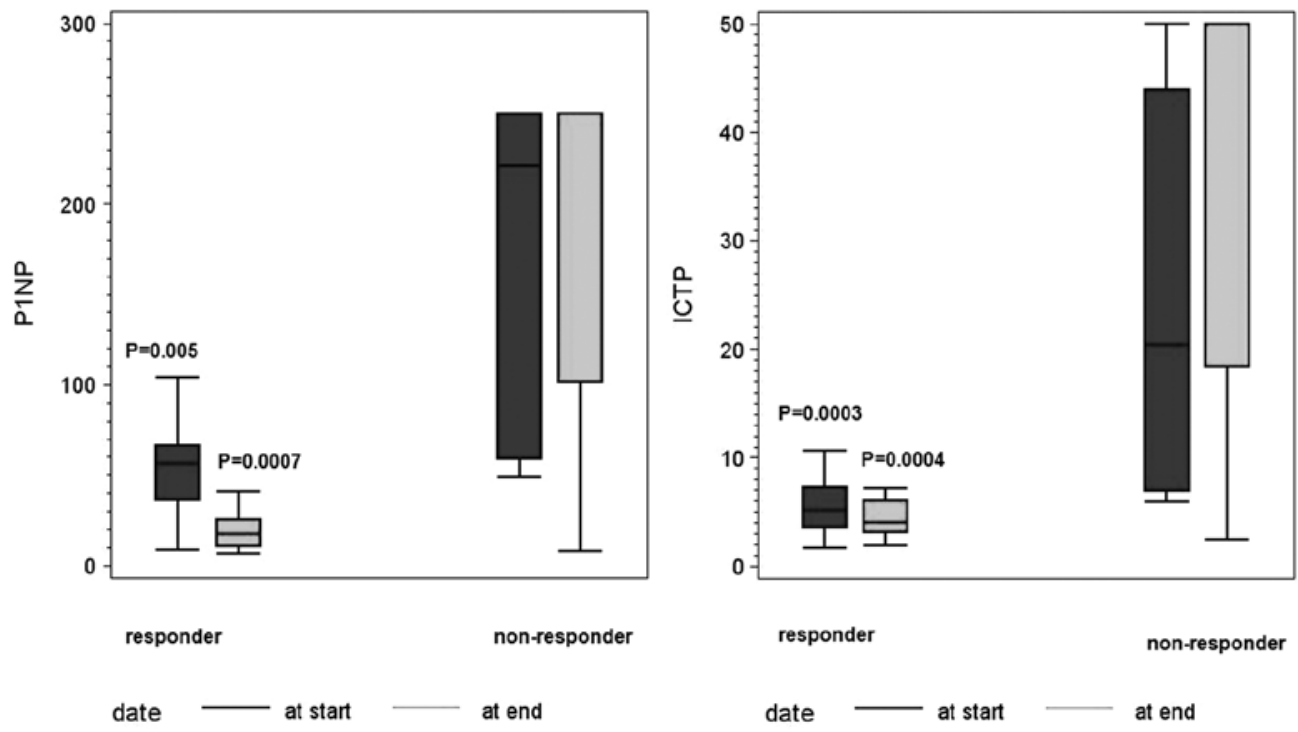

Figure 2. Correlation of levels of PINP/ICTP and clinical response.

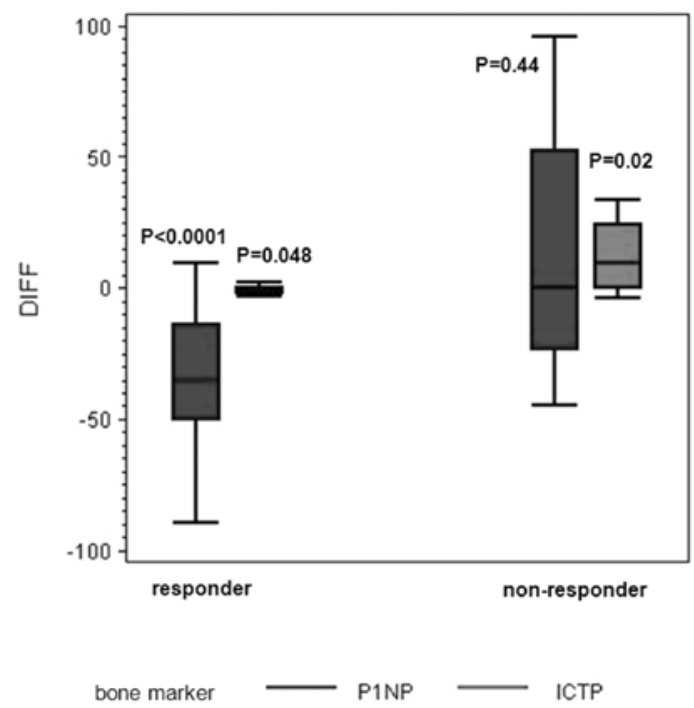

Figure 3. Differences (values at end-start) in levels of PINP and ICTP. noted when tumor marker levels were analyzed with respect to the number of $\mathrm{BM}(\mathrm{CEA} \mathrm{P}=0.51$ and $\mathrm{CA} 15-3 \mathrm{P}=0.13)$. Median value of CEA for patients with $>3 \mathrm{BM}$ was $3.6 \mathrm{ng} / \mathrm{ml}$ (IQR 1.6-7.3) and the median value of CEA for patients with $\leq 3 \mathrm{BM}$ was $2.4 \mathrm{ng} / \mathrm{ml}$ (IQR 1.2-6.5). Median value of CA 15-3 for patients with $>3 \mathrm{BM}$ was $64 \mathrm{U} / \mathrm{ml}$ (IQR 21-160) vs. $19 \mathrm{U} / \mathrm{ml}$ (IQR 9-61) for patients with $\leq 3 \mathrm{BM}$.

Correlation between tumor markers and response to therapy. Median value for CEA determined at the start of the therapy was $2.7 \mathrm{ng} / \mathrm{ml}$ (IQR 1.2-5.2) for responders and $11.7 \mathrm{ng} / \mathrm{ml}$ (IQR 2.2-57.1) for non-responders $(\mathrm{P}=0.039)$. The value for $\mathrm{CA} 15-3$ was 34.5 U/ml (IQR 16.0-94) for responders and 149 U/ml (IQR 85-698) for non-responders $(\mathrm{P}=0.035)$. The values determined at the end of the follow-up period were $2.5 \mathrm{ng} / \mathrm{ml}$ (IQR 1.5-4.6) for responders vs. $62.7 \mathrm{ng} / \mathrm{ml}$ (IQR 6-187) for non-responders for CEA and $27.5 \mathrm{U} / \mathrm{ml}$ (IQR 15-79) for responders vs. $603 \mathrm{U} /$ $\mathrm{ml}$ (IQR 102-6000) for non-responders for CA 15-3 ( $\mathrm{P}=0.002$ 
A
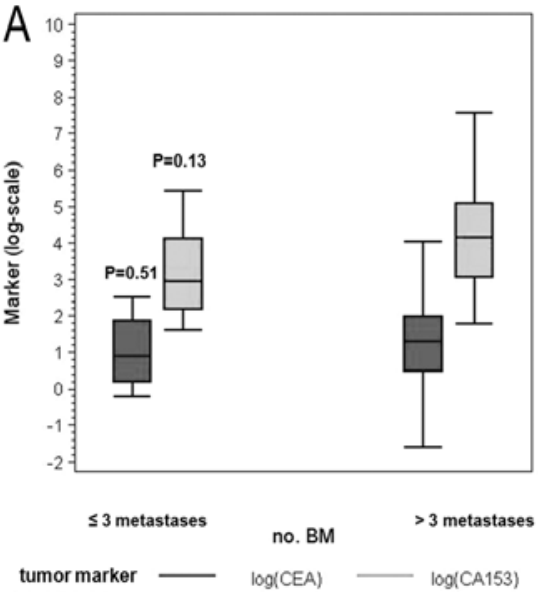

B

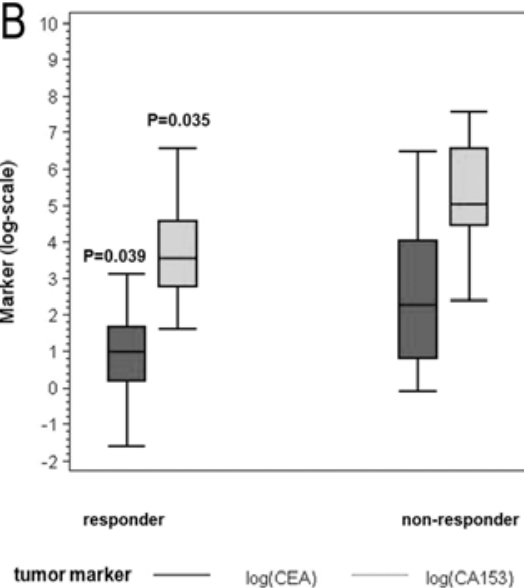

C

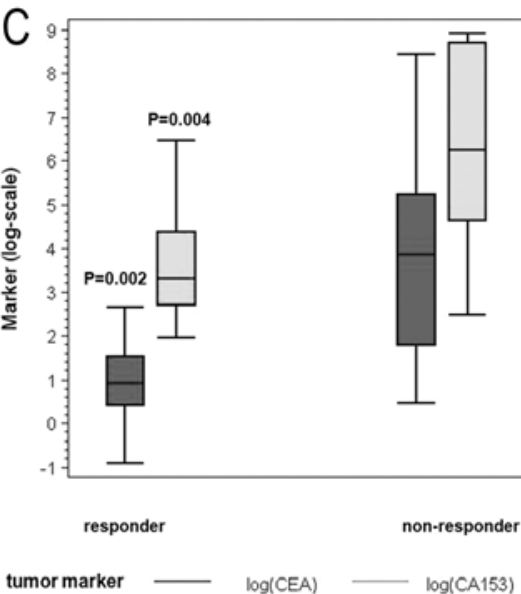

Figure 4. Prognostic values of tumor markers at the start of the study (A and B) and at the end of the study (C).

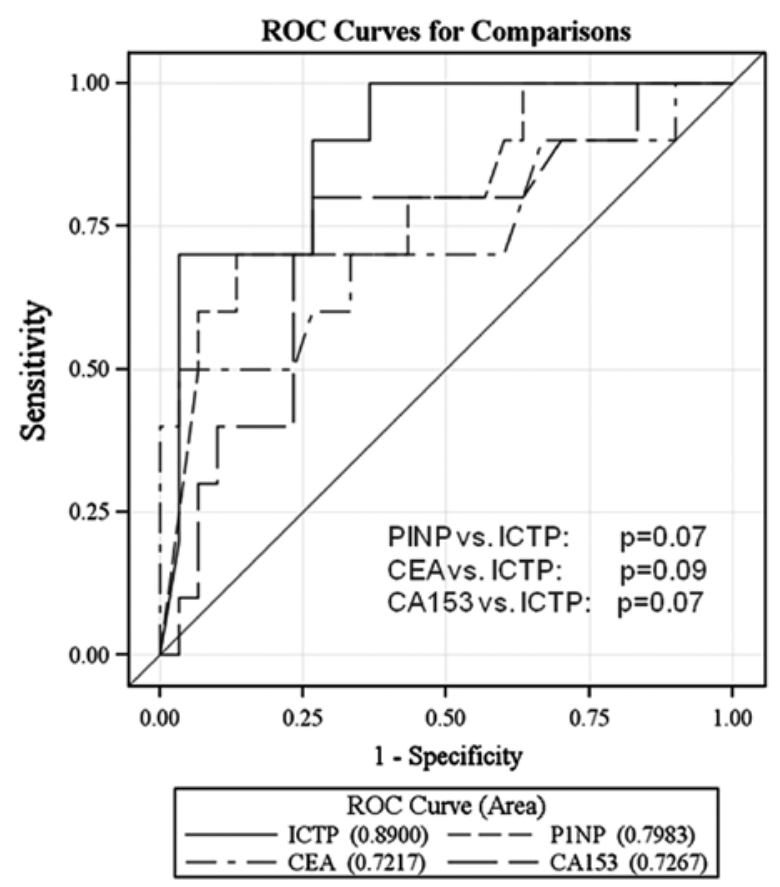

Figure 5. Receiver operating characteristic (ROC) curves comparing the discriminative power of the tested markers.

for CEA and $\mathrm{P}=0.004$ for CA 15-3). Tumor marker levels significantly distinguished responders from non-responders (Fig. 4B and C).

Comparison of markers of bone turnover and tumor markers. As shown in Fig. 5, ROC analyses demonstrated that measurement of PINP and ICTP revealed more specificity and sensitivity than the determination of CA 15-3 and CEA. Although statistical significance was not reached, we observed a tendency for best discrimination with ICTP (ICTP vs. PINP, $\mathrm{P}=0.07$; ICTP vs. CEA, $\mathrm{P}=0.09$ and ICTP vs. CA $15-3 ; \mathrm{P}=0.07$, respectively).

Correlation between the number of BM and response to therapy. Twenty-nine out of 40 patients $(72.5 \%)$ showed $>3$ BM confirmed by imaging and 11 out of 40 patients (27.5\%) presented with $\leq 3 \mathrm{BM}$. Thirty out of the 40 patients demonstrated response to therapy, while 10 out of 40 patients did not. All non-responders had $>3 \mathrm{BM}$ at baseline $(\mathrm{P}=0.04)$.

Correlation of the results with menopausal status. Thirty out of 40 patients were postmenopausal, 23 out of 30 were responders and 7 out of 30 non-responders, respectively. The premenopausal cohort showed 7 out of 10 responses and 3 out of 10 had no response, comparable to the postmenopausal group $(\mathrm{P}=0.69)$. While expression of the markers at the start of the study did not differ between the premenopausal and postmenopausal groups (data not shown), they were noted in the findings of the postmenopausal patients of the full study group, being higher non-responders. The median value of ICTP at the start of the study for the responders was 5.7 vs. $23.8 \mu \mathrm{g} / 1$ for non-responders. The median value of PINP was 63.4 vs. $192.0 \mu \mathrm{g} / \mathrm{l}$, respectively, with $\mathrm{P}=0.006$ for ICTP and $\mathrm{P}=0.04$ for PINP. For tumor markers, the median CEA was $2.8 \mathrm{ng} / \mathrm{ml}$ for responders and $18.2 \mathrm{ng} / \mathrm{ml}$ for non-responders $(\mathrm{P}=0.014)$ and the values for $\mathrm{CA} 15-3$ were $35 \mathrm{U} / \mathrm{ml}$ and $150 \mathrm{U} /$ $\mathrm{ml}$, respectively $(\mathrm{P}=0.08)$.

\section{Discussion}

Several studies have shown that the serum of patients with metastatic bone disease contains significantly higher concentrations of markers of bone resorption and formation than age-matched controls (12). In our study, patients with more than three involved sites of BM had significantly higher serum levels of PINP and ICTP than patients with three or fewer sites of involved skeletal metastases as compared to a group without any BM. Furthermore, we demonstrated a significant relationship between PINP, ICTP and clinical outcome. When patients were stratified into responders and non-responders, significant differences in the expression levels of the markers were obtained for both groups. Non-responders had significantly more than three BM. Comparing markers of bone turnover and tumor markers, with regard to the best diagnostic efficacy using ROC-analysis, ICTP and PINP revealed more specificity and sensitivity than CA 15-3 and CEA. No correlation between menopausal status and response to therapy was determined. 
At present, the detection and monitoring of $\mathrm{BM}$ relies on imaging studies such as bone scan, radiography, CT scan or MRT scan. Besides being limited by low sensitivity and specificity, bone scans may be unable to detect osteolytic metastasis and are affected by the flare phenomenon $(13,14)$. Furthermore, these imaging techniques are time-consuming and costly for patients. To date, none of the biochemical markers of bone turnover are able to monitor the development and progression of metastatic bone disease as accurately as do well-established bone imaging techniques (15). When used alone, they do not possess sufficient diagnostic and prognostic specificity to assess the overall treatment outcome for patients with cancer (16). However, in combination with other diagnostic techniques and prognostic markers, these biochemical markers of bone turnover may be useful tools in the assessment of cancer with metastatic bone disease (15-20).

In our study, levels of PINP and ICTP were elevated in patients with osseous MBC as compared to patients without bone involvement. Similar results were previously confirmed by Pollmann et al, who showed elevated PINP levels for osseous MBC patients (21). The quality of life of MBC patients could be preserved by determining the expression of bone marker levels before indicating imaging techniques to follow up therapeutic response. Furthermore, our data revealed that PINP and ICTP reflect the therapeutic response of BM. Thus, assessing serum PINP and ICTP may replace primary techniques in the future.

In our analyses, there was a strong relationship between serum levels of PINP and ICTP and the extent of BM but no association with the presence of visceral metastases only. Similar data were shown by Costa et al, who found an association between bone marker concentrations ( $\mathrm{N}$-telopeptides and bone alkaline phosphatase) and the extent of BM in cancer patients but not with the presence of extraskeletal metastases (22). Berruti et al offered another possible use of these markers in patients with metastatic bone disease, i.e., to assess both tumor burden and extent of true bone pain (23). In their retrospective study, they showed that markers of bone turnover were significantly associated with the extent of disease or tumor burden by demonstrating a relationship between the concentrations of markers (serum bone alkaline phosphatase, the C-terminal telopeptide of type I collagen, and urine deoxypyridinoline) and the number of skeletal sites involved (skull, spine, femur, ribs, pelvis, and others). The more skeletal involvement of the disease, the higher marker concentration they found (24). Our data define this association more precisely, showing that the serum marker expression levels of PINP and ICTP were significantly higher at baseline when more than three skeletal sites were involved.

Other reports have also shown that resorption and formation markers such as urinary pyridinoline (Py) and deoxypyridinoline (dPy) correlate with the number of lesions and/or the number of skeletal segments involved in patients with prostate cancer (25). Recent studies reported that bone resorption markers may detect an early progression in patients with stable disease in imaging studies $(26,27)$. Furthermore, a high level of biochemical markers as NTX (N-terminal telopeptide) have been associated with increased skeletal-related events, disease progression and death compared with normal NTX levels in patients with malignant skeletal metastases $(17,28)$. Our data confirm that there is a high evidence to suggest that ICTP and PINP can be used to determine the degree of skeletal involvement in MBC. In our analysis, PINP and ICTP demonstrated utility for the differentiation of patients with BM from patients without metastatic spread to the bone, while tumor marker expression was not as effective. Measurement of the biochemical markers to determine the extension of skeletal involvement could change and improve strategies in the clinical management of metastatic breast cancer. More than three BM correlated significantly with no response to therapy. High levels of PINP and ICTP at baseline or at any time during the course of the disease may indicate that more aggressive intervention strategies in addition to ZOL therapy are needed to prevent skeletal morbidity. It may be appropriate, therefore, for marker levels to be assessed at regular intervals during the course of metastatic bone disease. In our analysis, PINP and ICTP presented a relationship with clinical outcome in patients with osseous MBC.

In clinical practice, measurement of tumor markers such as CEA and CA 15-3 are commonly used for monitoring therapy response to a variety of breast cancer-related therapies. CA 15-3 appears to be more sensitive than CEA when the primary tumor is diagnosed and when metastasis is discovered. In this last situation, it allowed monitoring in two-thirds of the cases (29). Other studies recommend the combination of both markers to improve diagnostic sensitivity $(30,31)$. We demonstrated no correlation between tumor marker levels and extent of metastatic spread, particularly no correlation to the number of BM. In contrast, tumor marker levels significantly distinguished responders from non-responders during systemic therapy added to ZOL.

In conclusion, in contrast to serum tumor markers, the determination of levels of PINP and ICTP allows inferences in regards to the number of BM and appears to be a useful tool for monitoring metastatic breast cancer patients undergoing bisphosphonate therapy with ZOL for treatment of BM.

PINP and ICTP, as well as the number of BM were significantly associated with clinical outcome and therapeutic response. Thus, increased levels of PINP or ICTP may be an early marker for therapy failure in patients with MBC. This could provide an opportunity for early therapeutic management that could extend the overall survival of patients and provide meaningful information concerning patients with $\mathrm{MBC}$ in daily practice.

\section{Acknowledgements}

This study was supported by a research grant from Novartis Pharma GmbH, Germany. We thank Gisela Koestner and Jens Rasch for their excellent technical assistance.

\section{References}

1. Plebani M, Bernardi D, Zanninotto M, De Paoli M, Secchiero S and Sciacovelli L: New and traditional serum markers of bone metabolism in the detection of skeletal metastases. Clin Biochem 29: 67-72, 1996.

2. Rosenthal DI: Radiologic diagnosis of bone metastases. Cancer 80: 1595-1607, 1997.

3. Jung K, Lein M, Stephan C, Von HK, Semjonow A and Sinha P: Comparison of 10 serum bone turnover markers in prostate carcinoma patients with bone metastatic spread: diagnostic and prognostic implications. Int J Cancer 111: 783-791, 2004. 
4. O'Sullivan JM and Cook GJ: A review of the efficacy of bone scanning in prostate and breast cancer. Q J Nucl Med 46: 152-159, 2002.

5. Costa L, Demers LM, Gouveia-Oliveira A, Schaller J, Costa EB, Moura MC and Lipton A: Prospective evaluation of the peptide-bound collagen type I cross-links $\mathrm{N}$-telopeptide and $\mathrm{C}$-telopeptide in predicting bone metastases status. J Clin Oncol 20: 850-856, 2002.

6. Lipton A, Costa L, Ali S and Demers L: Use of markers of bone turnover for monitoring bone metastases and the response to therapy. Semin Oncol 28 (4 Suppl 11): 54-59, 2001.

7. Green JR: Bisphosphonates: preclinical review. Oncologist 9 (Suppl 4): 3-13, 2004.

8. Coleman RE: The clinical use of bone resorption markers in patients with malignant bone disease. Cancer 94: 2521-2533, 2001.

9. Roodman GD: Mechanisms of bone metastases. N Engl J Med 350: 1655-1664, 2004

10. Rosen LS, Gordon D, Kaminski M, Howell A, Belch A, Mackey J, Apffelstaedt J, Hussein MA, Coleman RE, Reitsma DJ, Chen BL and Seaman JJ: Long-term efficacy and safety of zoledronic acid in the treatment of skeletal metastases in patients with non-small cell lung carcinoma and other solid tumors: a randomized, phase III, double-blind, placebo-controlled trial. Cancer 98: 1735-1744, 2003.

11. Saad F, Gleason D, Murray R, Tchekmedyian S, Venner P, Lacombe L, Chin J, Vinholes J, Goas A, Zheng M and for the Zoledronic Acid Prostate Cancer Study Group: Long-term efficacy of zoledronic acid for the prevention of skeletal complications in patients with metastatic hormone-refractory prostate cancer. J Natl Cancer Inst 96: 879-882, 2004.

12. Pecherstorfer M, Zimmer-Roth I, Schilling T, Woitge HW, Schmidt H, Baumgartner G, et al: The diagnostic value of pyridinium cross-links of collagen, serum total alkaline phosphatase and urinary calcium excretion in neoplastic bone disease. J Clin Endocrinol Metab 80: 97-103, 1995.

13. Vogel CL, Schoenfelder J, Shemano I, Hayes DF and Gams RA: Worsening bone scan in the evaluation of antitumor response during hormonal therapy of breast cancer. J Clin Oncol 13: 1123-1128, 1995.

14. Pollen JF, Witztum KF and Ashburn WL: The flare phenomenon on radionuclide bone scan in metastatic prostate cancer. AJR Am J Roentgenol 142: 773-776, 1984.

15. Huang Q and Ouyang X: Biochemical-markers for the diagnosis of bone metastasis: a clinical review. Cancer Epidemiol 6: 94-98, 2011.

16. Seibel MJ: Clinical use of markers of bone turnover in metastatic bone disease. Nat Clin Pract Oncol 2: 504-517, 2005.

17. Coleman R, Brown J, Terpos E, Lipton A, Smith MR, Cook R and Major P: Bone markers and their prognostic value in metastatic bone disease: clinical evidence and future directions. Cancer Treat Rev 34: 629-639, 2008.

18. Seibel MJ: The use of molecular markers of bone turnover in the management of patients with metastatic bone disease. Clin Endocrinol 68: 839-849, 2008
19. Cremers S and Garnero P: Biochemical markers of bone turnover in the clinical development of drugs for osteoporosis and metastatic bone disease: potential uses and pitfalls. Drugs 66: 2031-2058, 2006

20. Brown JE, Cook RJ, Major P, Lipton A, Saad F, Smith M, Lee KA, Zheng M, Hei YJ and Colemann RE: Bone turnover markers as predictors of skeletal complications in prostate cancer, lung cancer, and other solid tumors. J Natl Cancer Inst 97: 59-69, 2005.

21. Pollmann D, Siepmann S, Geppert R, Wernecke KD, Possinger K and Lüftner D: The amino-terminal propeptide (PINP) of type I collagen is a clinically valid indicator of bone turnover and extent of metastatic spread in osseous metastatic breast cancer. Anticancer Res 27: 1853-1862, 2007.

22. Costa L, Demers LM, Speicher T, Gouveia Curley E, Harvey H, et al: Biochemical markers of bone turnover correlate with the extent of metastatic bone disease. American Society of Clinical Oncology 35th Annual Meeting, Philadelphia, 1999.

23. Berruti A, Dogliotti L, Gorzegno G, Torta M, Tampellini M, Tucci M, Cerutti S, Frezet MM, Stivanello M, Sacchetto G and Angeli A: Differential patterns of bone turnover in relation to bone pain and disease extent in bone in cancer patients with skeletal metastases. Clin Chem 45: 1240-1247, 1999.

24. Demers LM: Biochemical markers in the management of patients with metastatic bone disease. Clin Chem 45: 1131-1132, 1999.

25. Ikeda I, Miura T and Kondo I: Pyridinium cross-links as urinary markers of bone metastases in patients with prostate cancer. $\mathrm{Br}$ J Urol 77: 102-106, 1996.

26. Costa L, Demers LM, Gouveia A, Schalla J, Costa EB, Demoura MC and Lipton A: Correlation of urinary N-telopeptide levels with progression of bone metastases: a prospective study (Abstract 12). Cancer 88 (Suppl): 3094, 2000.

27. Zafeirakis A: Collagenous and non-collagenous biochemical markers of bone metastases from prostate cancer. Hippokratia 14: 164-169, 2010.

28. Lipton A, Cook R, Saad F, Major P, Garnero P, Terpos E, Brown JE and Coleman RE: Normalization of bone markers is associated with improved survival and elevated bone resorption receiving zoledronic acid. Cancer 113: 193-201, 2008.

29. Pons-Anicet DMF, Krebs BP, Mira R and Namer M: Value of CA 15:3 in the follow-up of breast cancer patients. Br J Cancer 55: 567-569, 1987.

30. Yildiz M, Oral B, Bozkurt M and Cobaner A: Relationship between bone scintigraphy and tumor markers in patients with breast cancer. Ann Nucl Med 18: 501-505, 2004.

31. Buamah PK, Bent DJ, Bodger WAH and Skillen AW: A profile of serum CA 15-3, carcinoembryonic antigen, alkaline phosphatase, and $\gamma$-glutamyl transferase levels in patients with breast cancer. J Surg Oncol 53: 84-87, 1993. 